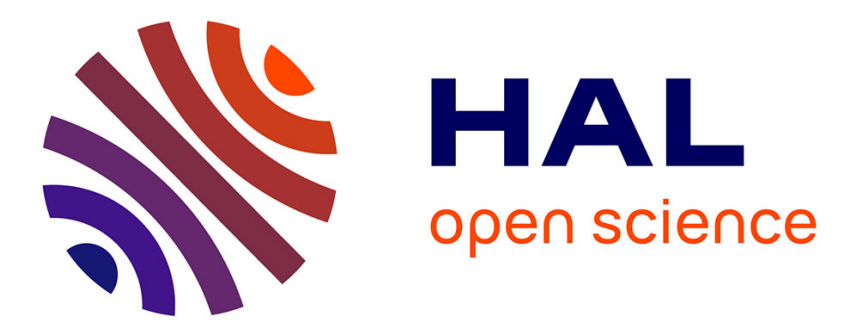

\title{
Maintenance activities scheduling under competencies constraints.
}

François Marmier, Christophe Varnier, Noureddine Zerhouni

\section{To cite this version:}

François Marmier, Christophe Varnier, Noureddine Zerhouni. Maintenance activities scheduling under competencies constraints.. IEEE International Conference on Service Systems and Service Management, IC SSSM'06., Oct 2006, Troyes, France. pp.1217-1222, 10.1109/ICSSSM.2006.320682 . hal00279894

\section{HAL Id: hal-00279894 \\ https://hal.science/hal-00279894}

Submitted on 15 May 2008

HAL is a multi-disciplinary open access archive for the deposit and dissemination of scientific research documents, whether they are published or not. The documents may come from teaching and research institutions in France or abroad, or from public or private research centers.
L'archive ouverte pluridisciplinaire HAL, est destinée au dépôt et à la diffusion de documents scientifiques de niveau recherche, publiés ou non, émanant des établissements d'enseignement et de recherche français ou étrangers, des laboratoires publics ou privés. 


\title{
Maintenance Activities Scheduling Under Competencies Constraints
}

\author{
François Marmier, Christophe Varnier, Noureddine Zerhouni \\ Laboratoire d'Automatique de Besançon (LAB) - UMR CNRS 6596 - ENSMM/UFC \\ 24, rue Alain Savary - 25000 Besançon - FRANCE
}

\{Fmarmier, Cvarnier, Zerhouni\} @ens2m.fr

\begin{abstract}
Competencies management in the industry is one of the most important keys in order to obtain good performance with production means. Especially in maintenance services field where the different practical knowledges or skills are their working tools. We propose here a methodology, which compares the human resource with parallel machine. As human resource competence levels of each are all different, they are considered like unrelated parallel machines. Our aim is to assign tasks to the adequate resources by minimizing time treatment for each task and the makespan.
\end{abstract}

Keywords: Competence, Contract, Human Resource, Maintenance, Scheduling, Unrelated Parallel Machine

\section{INTRODUCTION}

To stay competitive, companies must manage their costs as much as possible and optimize their production means operations. In order to support a better equipments' availability, and through them of the company, the maintenance service intervenes. It deals with problems before or after the breakdowns, at any place. This improvement mainly requires a better management of the workforce and their competencies.

It is not possible to determine precisely the necessary human resources number in a maintenance service [1]. Indeed, factors making possible to determine the adapted capacity are prone to uncertainties. Those are due to several parameters (variations of the intervention requests which are never similar, arrival dates of requests, requests' contents, necessary treatment duration and equipments availabilities as well as elements related to the interventions). Then, the different tasks are well known when they occur. The reactivity and the organization of the maintenance service will depend on the importance of the required treatment.

There are mainly two types of maintenance activities: The preventive maintenance, whose activities can be planned on long term, and the corrective maintenance which is related to the non foreseeable breakdowns. Within the service of maintenance, employees have different competencies and different qualification levels. Treatment speed and then the service reactivity will depend on the choice of the employees assigned to the task.

The goal of this management is to assign tasks to the bestknown resource. In this article, we detail a methodology which will allow us to assign tasks to resources by distributing the load between them. The rest of the paper is organized as followed: In the second section we will introduce how maintenance services can be managed. In the third part, we will present our scheduling problem. Then we develop our model and a resolution approach. Finally, we will discuss the different obtained results.

\section{MAINTENANCE MANAGEMENT}

There are various forms of management of maintenance. Indeed, if the company itself does not assume maintenance, this one can then be sub-contracted. The monitoring, the preventive and corrective maintenance can thus be entrusted directly to the manufacturer of the equipment (expert on this type of equipment) or with a company specialized in industrial maintenance (expert in monitoring and in remote maintenance field but general practitioner as for the monitored equipment). The equipment can also be rented, and if maintenance is not assumed by the user company, it can be sub-contracted too.

Our work is focused on the case of a multi-site company, in which, each site has its own maintenance service. One of these maintenance services has an increasing interest because it is responsible for particular interventions requiring important equipment or some more qualified staff. In this paper we will treat the maintenance activities scheduling in the dedicated maintenance service.

A maintenance service has to answer to its customers service demand. To do so, it disposes of human and material resources. Human resources are all different due to their qualification level in the required technical fields. Human resource being in limited quantity; the task's maximum number, which can be treated at the same time, is equal to the resource number. Depending on the resource choice for a task treatment, the treatment time (or the duration) of this-one will change. However, all the resources must be occupied. Then it will not necessary be the most efficient resource who will be assigned to the task's treatment. The tasks' assignment corresponds to a succession of tasks within human resources working time.

\subsection{Tasks management}

Equipment which has undergone some break-down stops has a decreased availability ratio. The difference between this rate and that contracted is tightened. In case of a new stop, the completion date of necessary intervention would be brought closer than for the previous stops. Availability becomes the first factor in the realization of a scheduling through the treatment completion date. 
In the literature, availability is known as temporal constraints [2] for the positioning of tasks at the time of the realization of a scheduling. This means that equipment is in fact occupied over certain periods by activities like maintenance [3], [4]. Unavailability is also related to the resources in order to mean that operators cannot work between certain dates [5]. To our knowledge, the concept of availability (or rather of equipment availability), is generally considered in the literature as a problem data. In our work, we considered it as an emergency indicator to assign priorities during the scheduling realization.

Equipment availability thus makes it possible to determine a temporal period, before the end of which the equipment must be operational. We obtain a completion date (a deadline) and also a period during which the treatment of the task must be carried out. A task with a very restricted treatment period will have priority on a task whose treatment can be delayed.

\subsection{Human resources management}

\section{Competencies management}

Boumane and al. [6] studied the different competency types which can be generic, and used in various professional situations, or specific to the activity. During her thesis, Agnès Letouzey carried out a study on nineteen companies to obtain their opinions on the operators' assignment problem [7]. It shows that operators' management, according to their competencies, is important for industry leaders and that there is still no software taking this into account. $79 \%$ of the companies think that operators' management is useful or essential in scheduling. Whereas in current softwares the operational duration is fixed, for the industrialist, the consideration of the operators' qualification is very important to determine their assignments, because, for them, the qualification level has (sometimes for $47 \%$ of them and always for $27 \%$ of them) an influence over the task's duration of realization. It appears the need for further development linking the competencies of human resources and the operational durations like in the determination of the potential of the company. Decisions are generally taken according to an operational level (what can do the best employees), rather than compared to a global vision of the workshop. This can be explained by the difficulties that $33 \%$ of them have to develop a strategy of assignment of the tasks to precise resources. There difficulties are explained by the fact that there are differences between each operator which are due to the history of each one. However, if the competencies levels of each one are known, it appears to be another problematic which is the workload balancing or the research of a compromise between the reactivity and the perturbation due to the modification of the employees planning. We consider that resources are working in parallel at the same time on different tasks and then our problem can be assimilated to a parallel machine problem.

\section{A parallel machine problem}

A maintenance service is an environment composed of $m$ operators working in parallel. They can all treat each task but there is not any proportionality notion between all the different treatment times. The resource which is the most effective for a task, would not necessary be effective for all tasks. The multiplicity of competencies shows that we have a parallel machine problem, but with unrelated machines which is noted $R$ or $R_{m}|\beta| \gamma$,[8] [9].

Pfund and al. presented a state of the art on the unrelated parallel-machine. One part is more precisely devoted to our problem: $R_{m} \| C_{\max }$ in the non-pre-emptive tasks cases [10]. Among all unrelated parallel-machine scheduling, problems, which aim is minimizing the makespan, are the most studied. Among the authors having worked on this subject, some of them developed approximate methods, with a fast execution but no optimal result. Ibarra and $a l$. then presented a methodology always used as comparison basis for current research in this field [11]. Their heuristic is based on a list algorithm and can lead to the worst case. Other authors developed exact methods which make possible to get the optimal solution. Mokotoff and Chrétienne presented results obtained using their exact cutting plane algorithm and compared it with the exact algorithms of Van de Velde and Martello [12], [13], [14].

\section{DESCRIPTION OF THE PROBLEM}

In scheduling and planning, the time horizon is often split in periods (the short, medium and long term). It allows time management and possibility for production problems modelling. One finds procedures using a slipping horizon into many studies of production planning [15], [16]. Then, we can study events on each time interval and not on a continuous scale of time. Once the completion date is determined for each task, the adequate period of insertion in the planning is deduced. The context of this article takes place in the medium term horizon.

In industrial context, the maintenance is generally perceived as constraint in a production task scheduling. In this approach, we consider that maintenance tasks are scheduled, instead of production tasks in classical problem. The manpower is then the limiting factor in the scheduling realization like the machines in a production scheduling study. Human resources are then organized in the maintenance service which has to plan their work.

\subsection{Equipment}

\section{Contracting and sub-contracting}

Within each plant, the maintenance service has to maintain equipment under operation. The level of the results to reach by the maintenance services is generally predetermined. In a subcontracting context, contracts signed between two (or more) partners which fixed their cooperation terms. In this case, a maintenance subcontractor and a company needing a maintenance service are concerned.

Contracts are will-intended agreements to produce law effects, between at least two partners.

Through them, we obtain different informations like availability of a concerning contracted equipment. When there is a breakdown, or, if for any reason equipment is stopped, it is always urgent for the customer that the repair happens quickly. But for the sub-contractor, regarding the global availability of the equipment, the maintenance has not necessarily to be immediate. 


\section{Equipment availability}

The guaranteed availability is a percentage of the opening time. Once the opening time for each equipment is defined, we obtain the real availability by the following formula:

$$
\text { Availability }(\%)=\frac{\text { Opening time }- \text { Maintenance Downtime }}{\text { Opening time }}
$$

The number and the nature of the equipment to be maintained are specified in the contract. In the case of workshop production including groups of similar equipments, it is possible to define an average availability ratio for the unit of these machines. Just like for a machine alone, this ratio will be measured and compared with the commitment taken for this group. The variations between the availability contracted and real availability of the different equipments makes it possible to deduce priority between different tasks.

The availability being defined by:

$A_{j}(t)$ the instantaneous availability of the equipment concerned by the task $j$ at the time $t$, with:

$$
0<A_{j}(t)<100 \%
$$

Where:

$$
A_{j}\left(t_{0}\right)=100 \%
$$

$A C_{j}$ the contracted availability for the equipment concerned by the task $j$, with: $0<A C_{j}<100 \%$

$A V_{j}(t)$ the availability variation between the contracted availability and the effective availability of the equipment

$$
A V_{j}(t)=A C_{j}-A_{j}(t)
$$

Then for the sub-contractor, we will speak of the criticality of intervention in order to avoid penalties of non-respect of the contract.

\section{Guaranteed availability and penalty}

Whereas the customer engages over the contract duration, the subcontractor guarantees an availability ratio. This one is located in a range of value (a class). If, for a machine or a group of machines, the objective of availability is not achieved, it will be considered as being in a lower class and a discount equivalent to the delta of the swing of class will be granted as a penalty by the service provider to the customer (Cost of corrective maintenance, preventive, and the level of spare parts target). Conditions concerning the penalties are defined while elaboration of the contract.

To calculate the level of availability which will be possibly guaranteed in the next contract, the subcontractor does not use the rate obtained the previous year but the one that would have being reached.

In the literature, many scheduling problems use penalties in the event of going beyond of the fixed dates. It is the case in particular in the scheduling problems of several tasks having a common completion date on a single machine. The objective of that kind of problem is known as the "earliness/tardiness problem". Only one task can be finished exactly for this completion date, others are finished before and have an advance penalty, or after and have a delay penalty [17]. Scheduling problems of tasks having a common completion date were shown as being NP-complete. (The optimal solutions cannot thus be easily obtained in case of a big size problem [18]). However a human resources management considering the skills levels of each operator in each competence will allow a better management of each task's duration and then the planning total duration.

\subsection{Tasks}

On medium-term, the maintenance service has to plan and assign the best human resource for the treatment of the different maintenance tasks. Preventive and conditional maintenances have for parameters a known duration, a starting date: $r_{i}$ (it cannot begin earlier) and a completion date: $d_{i}$ (it has to be completed before). Concerning the corrective maintenance tasks, they also have a estimated duration, dependant on a correct diagnosis. Their $r_{i}$ date is necessarily the present date because maintenance is required only when breakdown occurs. But the real beginning date of treatment can be later in case of replacement component shortage. The due date is known like for the preventive tasks. This allows using the same modelling for the different kind of tasks. The task $j$ is composed by a basic treatment time $p_{j}$ and the type of competence which is necessary to work on $(j=1 \ldots n)$. Tasks are distinguished between each others by a competence required to each resource, to be able to treat them. For example, the competence could be mechanic, electricity, automation or a certification. But the effective duration the task $j$ will be dependant of the resource who will have to treat it.

\subsection{Human resources}

The maintenance service is composed by $m$ human resources $(i=1 \ldots m)$, characterized by a competence profile. Relative speeds do not depend only on the tasks. Each resource has a corresponding qualification level for each task and operators will treat them more or less quickly. The duration of the job $j$, by the human resource $i$ is denoted by $p_{i j}$. With:

$$
p_{i j}=f\left(p_{j}, C_{i j}\right), \forall i \in\{1, \ldots, m\}
$$

Where $C_{i j}$ is the competence rate of resource $i$ in the competence which is used to achieve the task $j$. It can be represented with a matrix in which, for each different kind of job, the rate corresponding to the required competence can be found.

$$
\left[\begin{array}{ccccc}
C_{1,1} & \cdots & C_{1, j} & \cdots & C_{1, n} \\
\vdots & \ddots & & & \vdots \\
C_{i, 1} & & C_{i, j} & & C_{i, n} \\
\vdots & & & \ddots & \vdots \\
C_{m, 1} & \cdots & C_{m, j} & \cdots & C_{m, n}
\end{array}\right]
$$

The treatment duration of two different tasks by two different resources enable observing that for one kind of task, a resource can be more powerful than one other, whereas, for the second task, it is the second one which is the most effective. 


\section{MODEL}

\subsection{Data}

$P_{j} \quad$ Penalties which could be obtain if the treatment of the task $j$ is not realized on time,

$C r_{j, t}=f\left(A V, P_{j}\right) \quad$ Criticality of the maintenance task $j$, at the time $t$,

$d_{j}=g\left(C r_{j}\right) \quad$ Due date of the task $j$,

$\bar{d}_{j}=\max \left(d_{j}\right) \quad$ Deadline of the task $j$.

The horizon considered is the duration specified in the contract, on which the availability is guaranteed (in general, one year duration after the opening time of the equipment). So, this duration is to $100 \%$ of the time. A stop of $1 \%$ of opening time, during the year, on equipment will make lose $1 \%$ of its availability.

\section{Variables}

$t_{j} \quad$ Planned date of the task $j$ (its beginning date),

$x_{i j} \quad$ Indicator of the tasks assignment. $x_{i j}=1$ if the task $j$ is assigned to a resource $i$, else $x_{i j}=0$.

\subsection{Constraints}

- Each task has to be assigned only once to only one resource:

$$
\sum_{j=1}^{n} x_{i j}=1, \forall i \in\{1, \ldots, m\}
$$

- Release date of the equipment:

$$
\forall j, t_{j} \geqslant r_{i}
$$

A task $j$ cannot be planned before the equipment $i$ is available.

- Due date:

$$
\forall j, t_{j}+p_{i, j} \leqslant \bar{d}_{j}
$$

with:

$$
d_{j} \leqslant \bar{d}_{j}
$$

$j$ should be compulsorily treated before its deadline $\bar{d}_{j}$ but it would be preferable finishing the task before its expiration date $d_{j}$. Then, $d_{j}$ will be used in order to determine the objective function.

\subsection{Objectives}

In order to respect contracts, treatment delays have to be minimized. For an efficiency reason, tasks have to be assigned to good resources. Then we have minimized the treatment time for each tasks and the makespan. In the next part, we will develop an algorithm to minimize the $C_{\max }$.

\section{PROBLEM RESOLUTION}

\subsection{Lower Bound}

We used the lower bound which is the simplest limit for a problem as $R_{m} \| C_{\max }$ and that one finds in particular in work of Ibarra and al. or of Mokotoff and Chrétienne. It consists in taking for each of the $n$ tasks, the most powerful of the $m$ resource and to deduce the shortest duration $p_{i j}$ for each task. Then we obtain for Lower Bound:

$$
L B\left(C_{\max }\right)=\max \left\{\left\lceil\frac{1}{m} \sum_{i=1}^{n} p_{i}^{\min }\right\rceil ; \max _{i \in\{1, \ldots, m\}} p_{i}^{\min }\right\}
$$

with:

$$
p_{j}^{\min }=\min p_{i j}, j \in\{1, \ldots, n\}
$$

\subsection{Assignment algorithm}

$\mathrm{L}=\left\{\right.$ tasks order by decreasing longest duration $\left.p_{i j}\right\}$; $\overline{\mathrm{L}}=\emptyset$;

While $(\mathrm{L} \neq \emptyset)$ Then

$\mathrm{k} \leftarrow$ first task of $\mathrm{L}$;

$\mathrm{i} \leftarrow$ fastest resource for process task $\mathrm{k}$;

If $\left(\sum_{j \in \bar{L}} p_{i j} x_{i j}+p_{i k} \leqslant \mathrm{LB}\right)$ then

$x_{i k} \leftarrow 1 ; x_{a k} \leftarrow 0$, for $a=1 \ldots n$ and $a \neq i$;

$\overline{\mathrm{L}} \leftarrow \overline{\mathrm{L}}+k ; \mathrm{L} \leftarrow \mathrm{L}-k$;

else

try to assigned task $k$ to the fastest resource $l$, with $l=1 \ldots n$ and $l \neq$ worst case that respect $\sum_{j \in \bar{L}} p_{i j} x_{i j}+p_{i k} \leqslant \mathrm{LB}$;

If ( $l$ not found) then

find resource $l$ so that: $\min _{l=1 \ldots n} \sum_{j \in \bar{L}} p_{l j} x_{l j}+p_{l k}$ If $(l=$ worst case $)$ then | Exception Algo. 2; end If

end If

$x_{l k} \leftarrow 1 ; x_{a k} \leftarrow 0$, for $a=1 \ldots n$ and $a \neq l$;

$\overline{\mathrm{L}} \leftarrow \overline{\mathrm{L}}+k ; \mathrm{L} \leftarrow \mathrm{L}-k ;$

end If

end While

Algorithm 1: Main algorithm

Insert the first task of the list which would not be treated by the worst resource at the head of the list If (all tasks $\in \mathrm{L}$ check $p_{j}^{\max }=\max p_{i j}$ ) then

Then assign the tasks without being worried with the fact that it could be to the worst resource end If

Algorithm 2: Exception

Results of this algorithm are presented in the table 1.

\subsection{Tardiness penalties}

There are various methods to take into account the tardiness, which is the respect of the tasks due-dates. In the previous algorithm we worked on minimized the maximal 


\begin{tabular}{|c|c|c|c|c|c|c|c|}
\hline \multicolumn{2}{|c|}{ Our algorithm } & \multicolumn{3}{|c|}{ ECT } \\
\hline $\mathrm{m}$ & $\mathrm{n}$ & $\begin{array}{c}C_{\max } \\
(t . u .)\end{array}$ & $\mathrm{SD}$ & $\begin{array}{c}\text { time } \\
(m s)\end{array}$ & $\begin{array}{c}C_{\max } \\
(t . u .)\end{array}$ & SD & $\begin{array}{c}\text { time } \\
(m s)\end{array}$ \\
\hline $\mathbf{2}$ & 20 & 122 & 1.02 & 12.55 & 129.32 & 0.51 & 0.80 \\
& 30 & 185.87 & 0.57 & 11.65 & 190.79 & 0.45 & 1.50 \\
& 40 & 238.71 & 0.44 & 18.05 & 248.16 & 0.31 & 4.60 \\
& 50 & 303.96 & 0.4 & 19.45 & 316.2 & 0.43 & 1.55 \\
& 60 & 353.51 & 0.38 & 28.90 & 368.29 & 0.4 & 4.70 \\
& 80 & 481.82 & 0.41 & 32.75 & 503.84 & 0.42 & 6.90 \\
& 100 & 595.9 & 0.36 & 43.85 & 625.9 & 0.4 & 7.80 \\
& 200 & 1224.31 & 0.33 & 138.35 & 1280.24 & 0.22 & 32.20 \\
\hline $\mathbf{5}$ & 20 & 45.83 & 1.2 & 8.65 & 47.1 & 1.23 & 1.55 \\
& 30 & 67.75 & 0.96 & 10.15 & 70.15 & 0.72 & 2.35 \\
& 40 & 90.97 & 0.71 & 12.60 & 93.54 & 0.62 & 3.10 \\
& 50 & 115.24 & 0.67 & 18.80 & 120.67 & 0.54 & 2.35 \\
& 60 & 143.65 & 0.75 & 18.00 & 150.66 & 0.51 & 7.00 \\
& 80 & 186.37 & 0.55 & 23.50 & 200.02 & 0.51 & 10.05 \\
& 100 & 225.87 & 0.51 & 34.60 & 244.11 & 0.47 & 12.50 \\
& 200 & 445.91 & 0.5 & 83.60 & 486.9 & 0.42 & 36.75 \\
\hline $\mathbf{8}$ & 20 & 28 & 1.18 & 9.50 & 28.21 & 1.07 & 2.35 \\
& 30 & 41.91 & 1.15 & 10.20 & 42.74 & 0.87 & 0.80 \\
& 40 & 54.67 & 0.72 & 18.70 & 56.06 & 0.64 & 3.20 \\
& 50 & 69.57 & 0.69 & 13.25 & 71.72 & 0.6 & 5.35 \\
& 60 & 82.68 & 0.58 & 15.65 & 86.1 & 0.56 & 7.00 \\
& 80 & 110.67 & 0.55 & 20.45 & 115.75 & 0.48 & 9.40 \\
& 100 & 139.56 & 0.56 & 36.05 & 147.65 & 0.5 & 14.85 \\
& 200 & 268.77 & 0.42 & 79.85 & 291.87 & 0.45 & 43.70 \\
\hline \multirow{6}{*}{} & & & & & &
\end{tabular}

Tab. 1: Results of our and of the ECT algorithm

completion time $\left(C_{\max }\right)$. We compared results already obtained, reorganized with an Earliest Due Date (EDD) posttreatment within each resource solution, with two others possibility.

To consider the tardiness, we replaced the pre-treatment with fixed tasks by decreasing longest duration, by an other with sorted tasks by increasing due-date $d_{j}$ (EDD). In order to take into account the potential penalties of each late task, we tried also a Weighted Shortest Processing Time first (WSPT) pre-treatment which sort tasks by their decreasing $w_{i} / p_{i}$

During the assignment part, the sorting of the tasks can be spoiled. To avoid this effect, we placed an EDD posttreatment sort for each resource assignment. Results of this adaptation are presented in the table 2, where H-EDD means our heuristic followed by an EDD post-treatment, EDD-H-EDD means that the Longest Processing Time (LPT) pre-treatment is replaced an EDD one and finally WSPT-H-EDD means that the pre-treatment is a WSPT one.

\begin{tabular}{|c|c|c|c|c|}
\hline \multicolumn{2}{|c|}{} & Low load & Medium Load & High Load \\
\hline H-EDD & $\sum U_{i}$ & 3 & 22 & 148 \\
& $\sum w_{i} T_{i}$ & 173 & 1141 & 7301 \\
& $C_{\max }$ & 448 & 464 & 461 \\
\hline EDD-H-EDD & $\sum U_{i}$ & 33 & 71 & 136 \\
& $\sum w_{i} T_{i}$ & 1656 & 3487 & 6841 \\
& $C_{\max }$ & 456 & 473 & 468 \\
\hline WSPT-H-EDD & $\sum U_{i}$ & 3 & 21 & 143 \\
& $\sum w_{i} T_{i}$ & 162 & 1027 & 7142 \\
& $C_{\max }$ & 454 & 469 & 466 \\
\hline
\end{tabular}

Tab. 2: Tardiness consideration

\section{COMPUTATIONAL RESULTS}

\subsection{Data generation}

We choose to use an algorithm like Ibarra's one (previously described), and to improve it for our problem[11]. This algorithm is called ECT: Earliest Completion Time. Results obtained by our algorithm and by the Ibarra's one are presented in table 1 . These averages have been computed over 20 problem instances. The numbers of tasks $(n)$ and maintenance operators $(m)$ have been chosen to be representative from the reality. The $C_{\max }$ columns contain the makespan obtained with both algorithms (in Time Unit). SD columns means Standard Deviation between the duration of affectations of the different operators in each solutions. The last column show the averaged time per solution. We carried out a computational experiment on a Pentium IV $3.00 \mathrm{GHz}$ considering tests obtained by generating randomly the $p_{i j}$ values. $p_{i j}$ values are principally obtained by the combination of the basic tasks' duration which is an integer from the uniform distribution $[1,16]$. This duration is multiplied by the competence level of the resource in the corresponding competence. For each task, a corresponding competence is determined by an integer from the uniform distribution [1 , 3]. It refers for each resource to a level, which is a real from the uniform distribution [1.01, 2.00], in this competence. This data are determined before the simulation. Considering the resources and tasks number, the complexity is then $O(n * m)$. Penalties are determined as integers from the uniform distribution $[1,100]$. They are assigned if the task treatment is finished after its duedate, which is also obtained following a uniform distribution. We used the algorithm in three cases: in case of low, medium and high load. These conditions are determined by the generation of the due-dates. For a same task and resource number, we create tasks due-dates in a nearer future. In order to ensure that each task could be finished in time (depending of the scheduling), their due-date cannot be fixed before $t=$ now and $t_{2}=$ now $+2 * p_{j}$ ("now" being the program launching date, in second). To regulate the load we modified the maximal limit value $t_{3}$ and then we obtained due-dates as reels from the uniform distribution $\left[t_{2}, t_{3}\right]$. For the low load case $t_{3}=t_{2}+720 t$.u., in the medium load case: $t_{3}=t_{2}+540$ t.u. and in the high load case: $t_{3}=t_{2}+360$ t.u. .

\section{Assignment algorithm}

The standard deviation (SD) allows knowing, for one same set of data, the load of each resource is close to the $C_{\max }$. In the case of an identical $C_{\max }$ for two different simulations: the bigger SD is, the more the operators (not concerned by the $C_{\max }$ duration) have free time for eventually new tasks. This problem is not a research of optimal but of good (figure 1) and fast answer. This solution will be complete by other treatments which will need also computation time. Then, we drove our research towards an heuristic. The lower bound (LB) used in the algorithm is not the best lower bound that could be used, because it is only reachable in certain rare and particular cases. A better lower bound would be globally the highest. However the maximal variation between LB and our solution varies only from $5 \%$ for a two resources and twenty tasks problem to $12 \%$ for an eight resources and two hundred tasks problem. This heuristic presents also an improvement of 


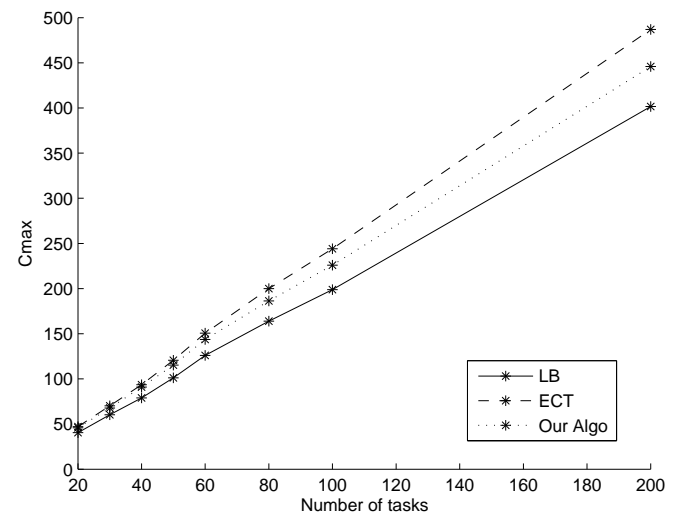

Fig. 1: Evolution of the $C_{\max }$ on 5 human resources

$8 \%$ compared to ECT for the eight resources and two hundred tasks problem, which is a large size problem. This is logic because of the added treatment of this algorithm. That treatment time is a little bit increased with our algorithm, but this is not perceptible for the program user, such it does not represent a problem.

\section{Tardiness}

The H-EDD heuristic allows us to obtain the best results concerning the $C_{\max }$ minimization. In the low and medium load cases, the WSPT-H-EDD heuristic presents the best results concerning the late tasks number and the penalties total, whereas, with a high load, best results are given by the EDD-H-EDD.

\section{CONCLUSIONS}

As already mentioned, this work allows assigning tasks to maintenance operators under skill constraint, minimizing the makespan. It is realized for the tasks which are in the medium-term horizon before each shift of the horizon. A good maintenance workforce plan considers each operator and its owned competencies in order to determine the strategy for the whole resources. We presented here some of the numerical results obtained. They were quickly obtained and near the optimal. We considered also the tardiness tasks number and the tardiness penalties by using different list treatment.

The next step has to take care of the earliness with release dates. Contrary to this tasks assignment experiments where maintenance operators are always available, tasks will have to be planned considering the operators time-table. Then we will have to work on an assignment problem under availability constraint.

\section{REFERENCES}

[1] E. Mjema, "An analysis of personnel capacity requirement in the maintenance departement by using a simulation method," Journal of Quality in Maintenance Engineering, vol. 8, no. 3, pp. 253-273, 2002.

[2] P. Baptiste, J. Carlier, and A. Jouglet, "Minimiser la somme des retards sur une machine avec dates de disponibilité," in MOSIM, 2001.

[3] R. Aggoune, "Job shop à deux jobs avec prise en compte de contraintes de disponibilité des machines," in MOSIM, 2003.

[4] G.-J. Sheen and L.-W. Liao, "Scheduling machinedependent jobs to minimize lateness on machines with identical speed under availability constraints," in Computers \& Operations Research, 2005.

[5] K.-P. Adzakpa and K.-H. Adjallah, "Minimisation du temps de séjour pondéré avec dates de disponibilité inégale des tâches : application à la maintenance," in MOSIM, 2003.

[6] A. Boumane, A. Talbi, D. Bouami, and C. Tahon, "Contribution méthodologique à la construction d'un référentiel de compétences en maintenance industrielle," in Colloque Francophone sur le thème : Performances et Nouvelles Technologies en Maintenance, 2003.

[7] A. Letouzey, "Ordonnancement interactif basé sur des indicateurs : Application à la gestion de commandes incertaines et à l'affectation des opérateurs," Ph.D. dissertation, Institut National Polytechnique de Toulouse, 2001.

[8] M. Pinedo, Scheduling, Theory, Algorithms and Systems. Prentice, 1995.

[9] P. Baptiste, E. Néron, and F. Sourd, Modèles et Algorithmes en Ordonnancement, Groupe GOThA, Ellipses, Ed., 2004.

[10] M. Pfund, J. W. Fowler, and J. N. D. Gupta, "A survey of algorithms for single and multi-objective unrelated machine deterministic scheduling problems," Journal of the Chinese Institute of Industrial Engineers, vol. 21, no. 3, pp. 230-241, 2004.

[11] O. Ibarra and C. Kim, "Heuristic algorithms for scheduling independent tasks of non identical processorsf," Journal of the Association for Computing Machinery, vol. 24, pp. 280-289, 1977.

[12] E. Mokotoff and P.Chrétienne, "A cutting plane algorithm for the unrelated parallel machine scheduling problem," European Journal of Operational Research, vol. 141, pp. 515-525, 2002.

[13] S. Martello, F. Soumis, and P. Toth, "Exact and approximation algorithms for makespan minimisation on unrelated parallel machines," Discrete Applied Mathematics, vol. 75, pp. 169-188, 1997.

[14] S. V. de Velde, "Duality-based algorithms for scheduling unrelated parallel machines," ORSA Journal of Computing, vol. 5, pp. 192-205, 1993.

[15] G. Hétreux, "Structures de décision multi-niveaux pour la planification de la production : robustesse et cohérence des décisions," Ph.D. dissertation, Institut National des Sciences Appliquées de Toulouse, 1996.

[16] G. Fontan, C. Mercé, and J. Erschler, Performance industrielle et gestion des flux. Lavoisier, 2001, ch. 3, pp. 69-111.

[17] D. Shabtay and G. Steiner, "Two due date assignment problems in scheduling a single machine," Operation Research Letters, 2006.

[18] S.-W. Lin, S.-Y. Chou, and K.-C. Ying, "A sequential exchange approach for minimizing earlinesstardiness penalties of single-machine scheduling with 
a common due date," European Journal of Operational Research, 2006. 